\title{
A Research on The information dissemination method of SI Design
}

\author{
Liu li-zhen \\ Wenzhou Vocational \& Technical College, Wenzhou. 325035 \\ Xia441@sina.com
}

Keywords: symbol coding; brand characteristics; information dissemination; store identity system

\begin{abstract}
Nowdays, Chinese business pay more attention to build their commercial Stores Identity System. In recent years, during the process of the " Second upgrade of corporate visual image", academic and business circles focus on how to express the inherent brand 's cultural concepts and national characteristic effectively, and how to reflects psychological need of the crowd accurately. use different levels of design symbols coding rules, and get rid of some unnecessary information. Finally they will find the distinctive characteristics of the corporate brand image to enhance the brand culture and competitiveness.
\end{abstract}

\section{Explore how to spread the space image with brand differences in the design methodology}

Based on years of practical experience, the author thinks that, in the process of national or brand characteristic symbol design, which means the encoding process of the symbol spreading, has a certain scientific laws and specific method. First in terms of methodology, we should realize clearly, in both the expression and the choice of construction materials, should be subject to the basic requirement of modern design theory and information communication, only in this way can get the accurate good symbol recognition and information arrival rate. Not split using some traditional Chinese symbols, but conformity the symbol by contemporary audience allow forms, instead of a variety of symbol of national culture, mining the most representative code combination according to scientific research, And spread these symbols according to the function different communication theory in a planned way hierarchical, is not only use ancestor or an area commonly used construction materials and technology as the carrier of the dissemination of information, but as far as possible use the most common material on the market at present, or the most easy to implement process the information. So no matter how the nationalization of the characteristic design, to meet the basic requirements of commercial space SI system- Identify effective, moderate volume, no noise interference, convenient construction simple, easy to copy. As Mr LiZhong hair says in the book < design and informatics $>$ : A qualified modern designers should have three kinds of abilities-1 information understanding and application 2 constitute ability of design modelling and color 3 convey information and the ability to communicate through design. The three abilities actually represents the information of import and integration ability, design codes and performance ability, symbol of output control and message control ability.

\section{Information coding level and rule of the difference brand space system}

According to the basic model of information communication of Shannon and Weaver., the communication process of a complete design information should abide by the following rules: From the information source(designer) get the information encoded(The design process) and then transformed into symbols or symbols groups(information)the symbol or information through certain information spreading media(channel) Interpretation by the sink(the information receiver), this process of interpretation symbols called decoding. In the links spreading channel, there is a noise interference problem, there are also symbol similar with spreading target symbolor confusing significance make the audience cannot accurately identify the symbol. So the accurate and effective coding link , control channel noise has become the key problem of this communication link which designers need to focus on. Ensure that the two links, the decoding process will fall into place, To 
ensure the communication intention understands by the recipient. In short, the encoding process need to be aware of three major problems: The first is disseminate information for different purposes - analysis what types of information propagation, and then to determine the type of information design and code in what order which is design program, the last is the type of information needed to follow those rules or methods in the process of coding which is the design method as figure1shows.

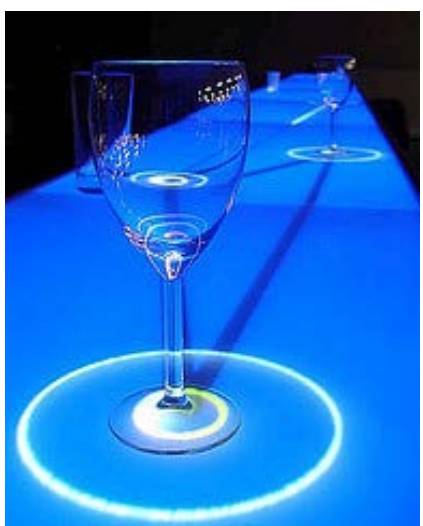

Fig 1 the symbol image

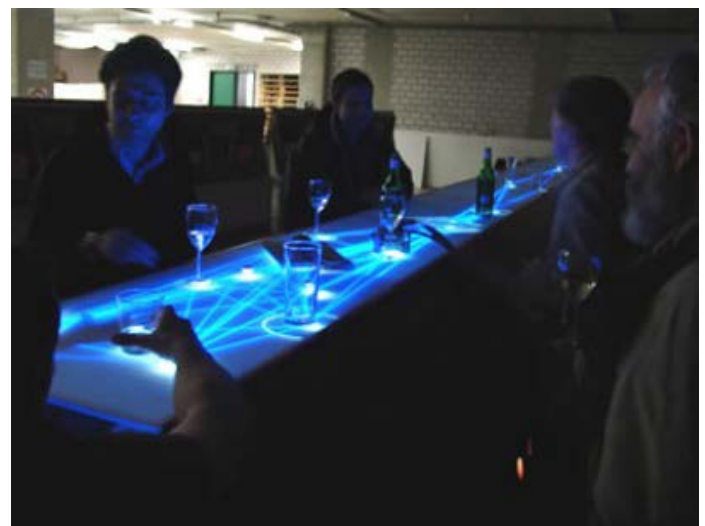

Figure 2 communication link

As the main brand's space recognition, brand channel store exists in various forms 、 business models and morphology, whether the brand interior stores in large shopping malls or similar kind of McDonald's outdoor shops on both sides of the street, whether it is as independent as Sinopec gas station chain form or large stores as Dazhong, Gome, as long as enterprises or brand after system of SI regulated, there are following five symbol characteristics in space system of communication channels in its business $1: 1$, logo and the word standard brands such as VI symbols2 performance style and modeling 3、 the standard store color 4、system 4 uniform material 5、The brand unique spatial aided shape。

In the multi view interpretation - Symbols - the designer, Symbolic interpretation's horizons fusion shows a multiple loop structure, that is take the interpreter and the designer as the main body, the former's attention is the interpreter perspective effect on the symbolic interpretation of behavior, the latter concern about the impact on the interpretation of the behavior of the designer perspective. As designers, we cannot control the decoding process, but can accurately interpretation symbols by careful arrangement of information and extensive research influence the information receiver, standing in the designer angle, the author believes that the above 5 big symbol feature groups can be divided into three sequentially coded information hierarchy according to their different purpose of propagation.

The first is the necessary information hierarchy, including the logo and the standard word etc. the core brand visual symbols. The so-called necessary information refers to is the information need to spread, like a personal understanding another person must first know its name. Company logo and the standard word are the external performance of the enterprise internal culture, industry, and other aspects of the intrinsic characteristics of the concept, the core of the visual image or text point directly to the connotation of enterprise. No matter what stores need to first communicated to consumers is name or logo. It is known as the necessary information.

Secondly the need to disseminate information hierarchy is homogeneous information hierarchy, the three main aspects of the hierarchical information including style and shape, store standard color system, unified material. The so-called homogeneous meaning is to code a dissemination of information and the same industry or the same population subdivision location nearest rival in all aspects of the requirements. This encoding is to ensure that the specification characteristics possible propagation of this industry in the practical application of SI space. After the study, different industries have different performance characteristics of the symbol, according to the features and the industry or the location of population subdivision in many industries tend to show a homogeneous rules. Take the color symbol as an example,the fast food industry such as McDonald's, KFC, pizza hut, real time, Yoshinoya etc. Volkswagen brand, its Wailimian main tone often focus on 
a narrow region in the orange to red the color phase ring. Its logo and standard word often use yellow and white and the background to achieve better contrast. In fruit time chain enterprises, Starbucks and many hot and cold drinks uses green as the main color, with white and eye-catching color as the standard word application. This is due to the difference of the decision of consumers on food's feelings, at the same time also affected by the construction materials, the color of the material can be obtained in all parts of the country, if in the coding process does not consider this kind of phenomenon, fast food restaurants designed with black or blue, consumers in the decoding process is bound to cause confusion or psychological uncomfortable feelings.

The user needs is to consider the problem from the user's point of view, describing the different user groups' requirements on product function, then we can make the demand of the user is divided into two categories :the spiritual needs and material needs. The spiritual demand is the psychological expectations of users in products, including product shape, color, human-computer interaction and so on; the material demand is the main feature of the performance of the products. Such as; the product structure, weight, volume, function and so on. In psychology we take the consumer demand for the purpose of need and the way to distinguish the need. Objective to need refers to the need to the final goal state, this kind of need is little, but very direct. Methods require refers to in order to achieve the purpose of chosen instruments, ways and means, it is for the purpose of serving the needs. We design the product is mainly in order to achieve the objective needs to make the way of objective reality of the need. A purpose of the needs of the consumers, often there will be much way need. For example: mobile is an objective need, how to move, what time, what way or what traffic tools for mobile and so on these is in order to achieve the purpose of moving to achieve way need.

Finally, the need to disseminate called feature information hierarchy, mainly refers to the brand's unique space or plane aided modeling. The auxiliary shapes similar plane auxiliary graphics in VI design, can be signs of evolution applied to spatial Wailimian construction shapes, and can also be some other form give according to the enterprise inner idea or brand, product unique characteristics. Such as McDonald's uncle figure in front of the McDonald's, children are very like, on the other hand to enrich the brand image, increase people's understanding of the connotation of the brand. Feature information level mainly point to competitors its enterprise unique dissemination, symbols or information others can not be copied, is the highest level of design and coding process. Ensure that the necessary information can be accurately convey the corporate name and other basic information; ensure the homogeneous information, can guarantee the accurate information to convey industry chain SI system of the enterprise, also conforms to the localization crowd the enterprises facing psychological needs, and to ensure that the special information, will create the only one SI system have the difference of identification, ensure the enterprise culture and brand image features and different from competitors. This information come from the other can level morphological evolution of symbols, also can undertake information mining from the unique one enterprise, brand, product, to find the most representative and a little convincing symbol encoding. As Adidas store commonly used three white line shape is according to its logo evolved, and represented the sports brand, linear speed and power. But this kind of modeling after the widely spreading, become the organic composition same with part store’s SI system.

\section{Channel noise control of brand space image information dissemination}

Information sign above three levels respectively, the dissemination of different functions, in order to ensure the information symbol is not interference we need know how to avoid noise in channel coding process. Generally speaking, the common channel noise are as follows:

1. Brand communication three levels symbols image not consistent with the positioning crowd's age, economic condition, social status.

2. The brands' performance of cultural background close with the form of other cultural backgrounds, improper handling will be very easy to let the consumer confusion by the decoding error.

3. Brand's cultural background, geographical characteristics, the characteristics of the product 
content is not familiar to consumers, and the choice of this kind of connotation as denotation means may not be most familiar to consumers, resulting in difficult decoding. Brand sense of color, style and even selection and industry characteristics of the materials do not meet, let people misunderstand the meaning of language, become a feature of other industries. If take the blue used in fast food restaurants, being mistaken for communication industry etc. In general the same industry with the same store SI language structure specification, also in the functional perspective, the same industry and in the same industry, such as furniture brand shops are mostly concentrated in large furniture stores, all belong to the glass facade, narrow head instore stores form, its style is bound by this form of constraint. If you ignore the industry, often in the message early produce very embarrassing recognition effect, such as a clothing store design into the cosmetics industry form, the selection of material, lighting configuration is not the same, can give a person the feeling of neither fish nor fowl. Construction materials LV stores'onsumption level、 decision modelling and selection of certain must different from Persia which is a popular brand.

4. The symbol information quantity(Assist other information, text information, color information) too much or too little, excessive information tend to caused decaying human memory, which is why modern design communication theory emphasizes the main problems concise. The color and text information quantity of iconic image is overmuch, seriously affecting the accurate dissemination of symbol and memory. Too little information may make people are difficult to decipher the full implication of the brand dissemination.

5. Brand standard word font or visual language modeling lines too thick, too small, word spacing properly, the volume is too large or too small to cause identification difficult, important problem which belongs to the ergonomics perspective effect recognition rate and recognition accuracy. For the recognition system, the choice of san serif a compact structure, simple and clear line is very important.

6. The color contrast is not loud, such as logo near the hue and the backplane or between lightness, resulting in a distance not effective identification

\section{Conclusion}

Along with the economic development and the rise of Chinese independent brands, the land situation of foreign brands rule all will change. Deep foundation Chinese culture determines the Chinese consumption habit and product preferences, this will also give the independent brand opportunities for development and living space. In the course of design, SI designers should not only sharp from the complex information to find the most valuable information for an enterprise to be integrated, to stand in design symbol science, information communication perspective treat design case, in all of the key links of the basic principles of the propagation process should not only focus on aesthetics, ergonomics, and according to the propagation and identification of repeated assessment of future promotion after the channel noise interference situation, the only way to become a qualified and wonderful SI system design.

\section{Reference}

[1] Li Zhong. Design of information science. Chengdu: Sichuan Fine Arts Publishing House [M], 2007

[2] Xia Zhi-Liang. Research on humanized design of Computer-aided Machinery and tools products[J] . Information Technology Journal， 2013,12(20);5935-5940

[3] Li Daoguo. Symbolic interpretation of the fusion of horizon [J]. Beijing: decoration, 2008 (11).

[4] (Germany) Andreas Beller, Gao Yi. The design of [M] oriented system. Beijing: Chinese Youth Press, 2008 